\title{
Design and Experimental Implementation of a Multi-Cloak Paraxial Optical System
}

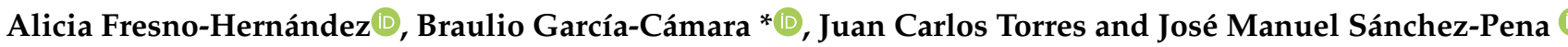 \\ Group of Displays and Photonic Applications, Carlos III University of Madrid, Avenida de la Universidad 30, \\ Leganés, E28911 Madrid, Spain; afresno@ing.uc3m.es (A.F.-H.); jctzafra@ing.uc3m.es (J.C.T.); \\ jmpena@ing.uc3m.es (J.M.S.-P.) \\ * Correspondence: brgarcia@ing.uc3m.es
}

\section{check for}

updates

Citation: Fresno-Hernández, A.; García-Cámara, B.; Torres, J.C.; Sánchez-Pena, J.M. Design and Experimental Implementation of a Multi-Cloak Paraxial Optical System. Photonics 2021, 8, 358. https:// doi.org/10.3390/photonics 8090358

Received: 20 July 2021

Accepted: 24 August 2021

Published: 28 August 2021

Publisher's Note: MDPI stays neutral with regard to jurisdictional claims in published maps and institutional affiliations.

Copyright: (c) 2021 by the authors. Licensee MDPI, Basel, Switzerland. This article is an open access article distributed under the terms and conditions of the Creative Commons Attribution (CC BY) license (https:/ / creativecommons.org/licenses/by/ $4.0 /)$.
Abstract: Electromagnetic cloaking has being continuously pursued using a large variety of approaches. In recent years, this effect has been observed using either complex devices based on the so-called Transformation Optics or simple systems based on conventional optics with proper characteristics. In the latter case, a simple arrangement of lenses working in the paraxial regime can provide broadband visible cloaking in a wide area. In this work, we analyzed and generalized this method by proposing a five-lens system producing at least three potential invisible regions with a large cloaked area ( $>90 \%$ of the visual field). In particular, we developed the mathematical formalism and show, both numerically and experimentally, the successful operation of the cloaking system with the naked eye.

Keywords: geometrical optics; optical cloaking; optical devices; optical imaging; ray tracing

\section{Introduction}

One of the most important magical artifacts of the wizarding world of Harry Potter is the invisibility cloak. This is because invisibility has been a fantasy, until recently. The advances on optical devices, imaging systems and light control make that electromagnetic cloaking can be real in a wide spectral range [1]. While the first modern attempts to achieve this phenomenon were based on imaging strategies [2], which displayed the environment behind the cloaked object, currently, research fields, including transformation optics [3], metamaterials [4] and nanophotonics [5], are able to produce electromagnetic cloaking in all spectral ranges.

The concept of transformations optics (TO), first developed by Pendry [6] and Leonhart [7], relies on the idea of manipulating the light around the cloaked object in such a way that the electromagnetic field before and after crossing the object is the same. This can be obtained through complex media capable of controlling light propagation. In recent years, metamaterials with this capability have been developed in the microwave [8-10], and the visible ranges [11-13].

Unfortunately, the experimental implementation of these techniques is not straightforward due to the large number of limitations. TO requires highly anisotropic metamaterials even though the invisibility is angular limited. Nevertheless, researchers are still working to overcome these constraints and create efficient cloaks [14,15]. In contrast to transformation optics and complex metamaterials, Choi and co-workers designed a simple optical system that provides invisibility in a certain plane [16].

In that work, the authors developed an innovative theoretical demonstration of optical invisibility in the paraxial regime. The optical system is composed of four common lenses in a configuration that resembles the the Köhler illumination in microscopes [17]. Using this, they proposed and experimentally demonstrated a ray optics cloak in the visible range for multidirectional angles in 3D. Its simplicity allows the system to be scaled to larger 
sizes, and the magnification is 1 . Nevertheless, its scalability to a higher number of lenses, and their invisible planes, is not straightforward.

While the former work is able to show a unique invisible plane, in the present work, we propose a simple system with five lenses that is able to produce three different invisible regions within the system, enlarging its potential application. To perform this, we developed a theoretical formalism to obtain the optimum relationships between the distances and focal lengths of the lenses and then numerically simulated it. Finally, we experimentally tested the system achieving three different invisible regions with a large area and for continuously multidirectional angles in 3D.

\section{Materials and Methods}

From a theoretical point of view, a perfect cloak could be defined as something completely hiding a certain volume, with a non-zero volume, and behaving as if its space was replaced by the surrounding medium. In principle, the satisfaction of these conditions is enough to achieve the invisibility of both the cloaked object and also the device producing that optical effect. Considering a simple optical system working in the paraxial approximation (small-angle approximation), the propagation of the light through the optical system can be described using ABCD matrices (also known as ray transfer matrices).

In this formalism, each optical element of the total system is described by a matrix, which operates on a vector describing the incoming light ray and allows the calculation of the outgoing light ray (defined by its transverse position and paraxial angle). Subsequently, the matrices of the different elements can be multiplied to obtain the ray transfer matrix of the whole optical system [18]. A perfect cloak does replicates the ambient medium throughout its volume, so that its ABCD matrix is a translation matrix with $t=L, L$ as the total length of the system, and $n_{t}=n=n^{\prime}, n$ as the refractive index of the surrounding medium, (assuming it as an uniform medium). As a result, the matrix of any perfect cloak should be the one shown in Equation (1).

$$
\left[\begin{array}{cc}
A & B \\
C & D
\end{array}\right]_{\text {perfect cloak }}=\left[\begin{array}{cc}
1 & \frac{L}{n} \\
0 & 1
\end{array}\right]
$$

This equation is a necessary condition for any paraxial cloak, also taking into account that the determinant of the ABCD matrices has to be 1 . These conditions can be satisfied by choosing the correct parameters of the optical system. In the particular case, we can control both the distances between the lenses and their focal lengths to reproduce these conditions and obtain invisibility.

This section comes to develop the theoretical formalism of the system shown in Figure 1 designing its geometrical parameters and predicting the invisible areas in the paraxial approximation. We consider five thin lenses in free space where the focal distances and the distances between them are analyzed to obtain space regions without rays, so any object in that regions is invisible. The refractive indices of the lenses, and the surrounding medium are considered homogeneous and isotropic. To treat this system mathematically, we use the $A B C D$ matrices formalism, knowing that [18]:

- The ABCD matrix of each thin lens is:

$$
S_{i}=\left[\begin{array}{ll}
A & B \\
C & D
\end{array}\right]_{\text {thin lens }}=\left[\begin{array}{cc}
1 & 0 \\
\frac{-1}{f_{i}} & 1
\end{array}\right]
$$

where $f_{i}$ is the focal length of each lens ( $i=1,2,3,4,5$ in this case).

- The ABCD matrix for the space between two lenses is only a translation matrix $M_{j}$ (see Equation (3)), considering free space $\left(n_{t}=1\right)$, and where $t=t_{j}$ is the distance between each two lenses $(j=1,2,3,4$ in this case). 


$$
M_{j}=\left[\begin{array}{cc}
1 & \frac{t_{j}}{n_{t}} \\
0 & 1
\end{array}\right]
$$

Then, multiplying the corresponding matrices, the complete system has an optical matrix like that one of Equation (4).

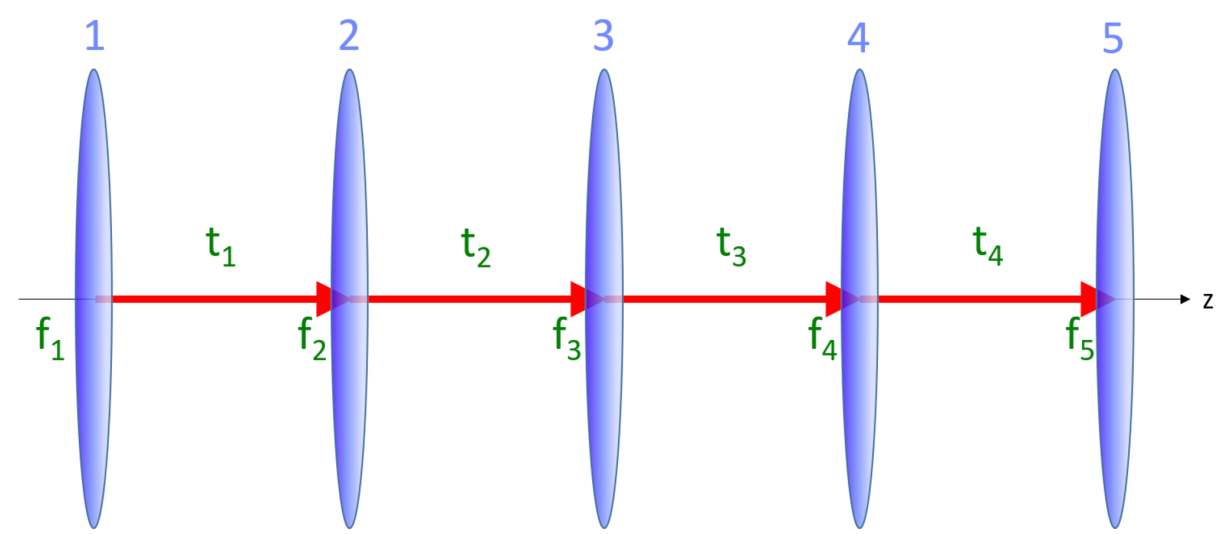

Figure 1. Simplified scheme for the five lenses system. $f_{i}$ is the focal distance of each lens, and $t_{i}$ the distances between two lenses.

$$
\left[\begin{array}{ll}
A & B \\
C & D
\end{array}\right]_{\substack{5_{\text {system }} \text { lenses } \\
\text { ste }}}=S_{5} \cdot M_{4} \cdot S_{4} \cdot M_{3} \cdot S_{3} \cdot M_{2} \cdot S_{2} \cdot M_{1} \cdot S_{1}
$$

To simplify the system and also make it unseen, it is considered symmetric. This means that the second half is equal to the first half. Mathematically, this can be done considering that $f_{1}=f_{5}, f_{2}=f_{4}, t_{1}=t_{4}, t_{2}=t_{3}$. Taking this into account and applying the cloaking conditions described before (in particular, we consider $A=1$ and $C=0$ ), we can obtain an expression of $t_{1}$ satisfying them.

$$
t_{1}=\frac{\left(f_{1} \cdot f_{2}+2 \cdot f_{1} \cdot f_{3}+2 \cdot f_{2} \cdot f_{3}-f_{1} \cdot t_{2}-f_{2} \cdot t_{2}\right)}{\left(f_{2}+2 \cdot f_{3}-t_{2}\right)}
$$

This Equation (5) can then be used to calculate the total length of the system $(L)$ as follows.

$$
L=2 \cdot t_{1}+2 \cdot t_{2}=\frac{2 \cdot\left(f_{1} \cdot f_{2}+2 \cdot f_{1} \cdot f_{3}+2 \cdot f_{2} \cdot f_{3}-f_{1} \cdot t_{2}+2 \cdot f_{3} \cdot t_{2}-t_{2}^{2}\right)}{f_{2}+2 \cdot f_{3}-t_{2}}
$$

At this point, and considering previous equations, the optical matrix was simplified like this:

$$
\left[\begin{array}{ll}
A & B \\
C & D
\end{array}\right]_{\substack{5_{\text {system }} \text { lesses } \\
0}}=\left[\begin{array}{cc}
1 & \frac{-\left(f_{1} \cdot\left(f_{1} \cdot f_{2}^{2}+2 \cdot f_{2}^{2} \cdot f_{3}+f_{1} \cdot t_{2}^{2}+2 \cdot f_{1} \cdot f_{2} \cdot f_{3}-2 \cdot f_{1} \cdot f_{2} \cdot t_{2}-2 \cdot f_{1} \cdot f_{3} \cdot t_{2}\right)\right)}{\left(f_{2}^{2} \cdot f_{3}\right)} \\
0 & 1
\end{array}\right]
$$

Now, we impose the last cloaking condition $B=L=2 t_{1}+2 t_{2}$ (using Equation (5) for $t_{1}$ ). This gives us the two following solutions for $f_{3}$ to obtain a perfect cloaking.

$$
f_{3_{1}}=\frac{f_{2}^{2} t_{2}^{2}-2 f_{1}^{2} f_{2}^{2}-2 f_{1}^{2} t_{2}^{2}-2 f_{1} f_{2}^{3}+f_{2}^{2} t_{2}\left(t_{2}^{2}+4 f_{1} t_{2}-4 f_{1} f_{2}\right)^{1 / 2}+2 f_{1} f_{2}^{2} t_{2}+4 f_{1}^{2} f_{2} t_{2}}{4\left(f_{1}+f_{2}\right)\left(f_{1} f_{2}-f_{1} t_{2}+f_{2} t_{2}+f_{2}^{2}\right)}
$$




$$
f_{3_{2}}=\frac{-\left(2 f_{1} f_{2}^{3}+2 f_{1}^{2} f_{2}^{2}+2 f_{1}^{2} t_{2}^{2}-f_{2}^{2} t_{2}^{2}+f_{2}^{2} t_{2}\left(t_{2}^{2}+4 f_{1} t_{2}-4 f_{1} f_{2}\right)^{1 / 2}-2 f_{1} f_{2}^{2} t_{2}-4 f_{1}^{2} f_{2} t_{2}\right)}{4\left(f_{1}+f_{2}\right)\left(f_{1} f_{2}-f_{1} t_{2}+f_{2} t_{2}+f_{2}^{2}\right)}
$$

In real experimental systems, the focal length of the different lenses is usually fixed, and what is required is to determine their positions in order to achieve invisible areas. This is $t_{1}=t_{4}$ and $t_{2}=t_{3}$. To do so, in a general case, the values of $f_{1}=f_{5}, f_{2}=f_{4}$ are substituted in Equations (8) and (9). This gives us to potential values of $f_{3}$ as a function of $t_{2}$. As the symmetry condition does not affect to lens 3 , we can decide a commercial value of the focal length, consequently determining the value of $t_{2}$.

The two potential values of $t_{2}$ from Equations (8) and (9) should be analyzed in order to evaluate its physical meaning. For instance, negative values are not valid, or there is no solution of the equation. Fortunately, in those cases the remaining solution is usually valid, and thus the system can still be resolved.

Once the value of $t_{2}$ is known, Equation (5) can be used to calculate $t_{1}$, and finally the total length of the system $(L)$. Lastly, the numerical values can be substituted in the optical matrix (Equation (4)) in order to check the fulfilment of the cloaking conditions.

\section{Results}

This section summarizes the main results that we obtained. To properly validate the proposed mathematical formalism, both numerical and experimental tests were performed using different values of focal lengths obtained from commercial lenses. In particular and for simplicity, in this work, we only focus our attention in the case of $f_{1}=f_{5}=200 \mathrm{~mm}$ and $f_{2}=f_{4}=75 \mathrm{~mm}$ and two different values of the third lens:

- $f_{3}=-150 \mathrm{~mm}$

- $f_{3}=-25 \mathrm{~mm}$

All these values correspond to commercial lenses from Thorlabs. Once these values are fixed, and considering the formalism described in the previous section, we can obtain the interlens distances satisfying the cloaking conditions. Once the main geometrical values were obtained, we tested them, first using a numerical model in Zemax and then an experimental implementation in the laboratory.

\subsection{Results for $f_{3}=-150 \mathrm{~mm}$}

In the present case, by applying our mathematical formalism, we obtained that $t_{1}=$ $t_{4}=290.1 \mathrm{~mm}$ and $t_{2}=t_{3}=147 \mathrm{~mm}$. Therefore, the total length of the system is $L=2 t_{1}+2 t_{2}=874.2 \mathrm{~mm}$.

Once the optical system was designed, its ray diagram was simulated with Zemax (see Figure 2a). Those planes with highest concentration of rays are the interesting ones because they correspond to the regions with the greatest area of invisibility. From now on, we use labels plane 1, plane 3, and plane 4 to refer them, as in Figure 2a. It is important to remark that we do not consider any plane in Zone 2, since there is no clear area of concentration of rays.

Figure $2 \mathrm{~b}$ shows the Spot Diagram at the interesting planes, calculated using the Zemax's Spot Diagram tool. The smallest diameter is placed in plane 1, and thus our best invisible region will be zone 1 , then zone 3 and finally zone 4 , as it can be seen from the results. This is because a larger light rays concentration means a larger invisible area in that plane. 


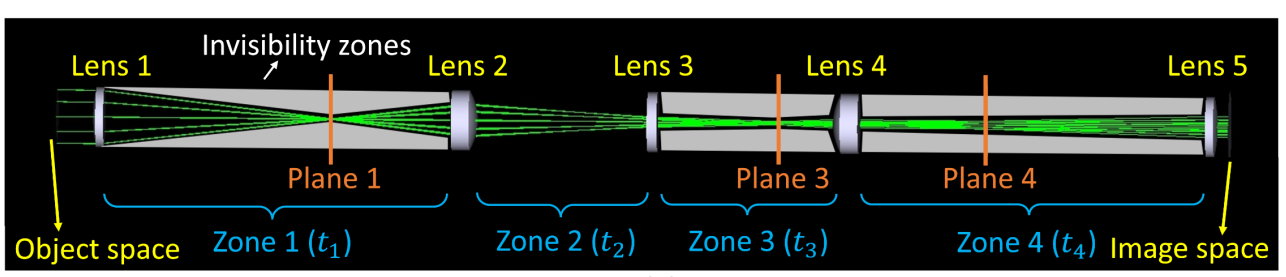

(a)

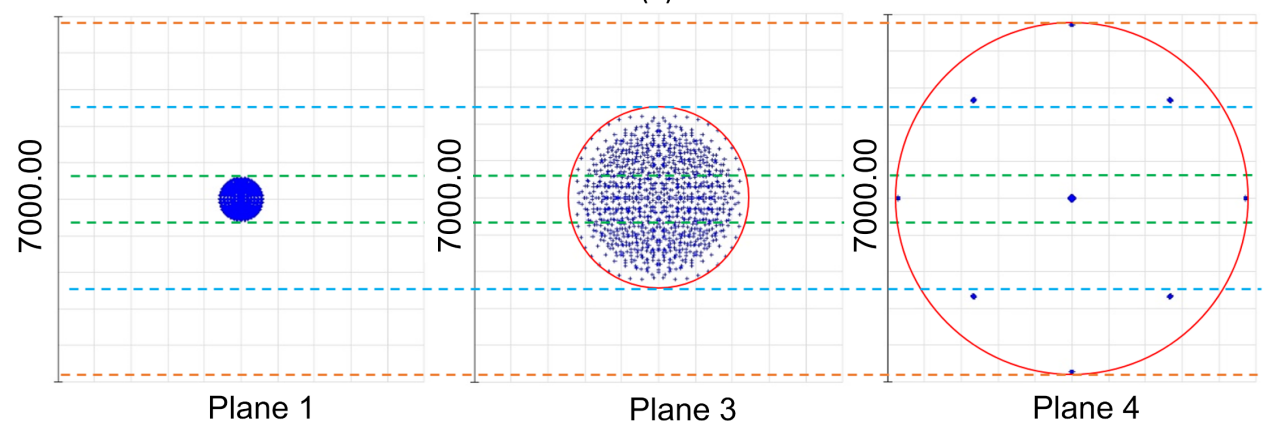

(b)

Figure 2. Simulation results of Case $f_{3}=-150 \mathrm{~mm}$. (a) Ray tracing for the case $f_{3}=-150 \mathrm{~mm}$. We can see the zones of invisibility and the planes used to calculate the diameter of the beam at the points of maximum invisibility. (b) Spot Diagram for the three planes of interest using the same scale to compare the sizes.

To numerically quantify the area of invisibility $\left(A_{i}\right)$, the area of the beam is subtracted from the visual area as in Equation (10), where $d_{i}$ is the diameter of the beam of rays on each plane while the diameter of visual region is equal to that of the lenses $(D=50.8 \mathrm{~mm}$ in our case).

$$
A_{i}=\pi\left(\frac{D}{2}\right)^{2}-\pi\left(\frac{d_{i}}{2}\right)^{2}
$$

As previously mentioned, we consider three planes of interest, this is $i=1,3,4$, and following the numerical results, we obtain that:

- $\quad$ Plane 1: $d_{1}=810.6 \mu \mathrm{m}=0.811 \mathrm{~mm} \rightarrow A_{1}=20.263 \mathrm{~cm}^{2}$

- $\quad$ Plane 3: $d_{3}=3384.77 \mu \mathrm{m}=3.385 \mathrm{~mm} \rightarrow A_{3}=20.178 \mathrm{~cm}^{2}$

- $\quad$ Plane 4: $d_{4}=6644 \mu \mathrm{m}=6.644 \mathrm{~mm} \rightarrow A_{4}=19.922 \mathrm{~cm}^{2}$

As it can be seen, although the largest invisible area is that corresponds to plane 1 with a $99.9 \%$ of the visual field, the invisibility regions are quite similar, with $99.5 \%$ and a $98.3 \%$ of the field in planes 2 and 3, respectively.

To experimentally verify these results, we set up the optical system shown in Figure $3 b$. The considered lenses are commercial ones with a $2^{\prime \prime}$ diameter $(D=50.8 \mathrm{~mm})$, which are mounted on an optical rail. These lenses have the required focal lengths, previously mentioned, being located at the corresponding distances between them. These distances and the position of the cloaked planes are described in the scheme of Figure 3a. The object, which will be cloaked, is a black ruler mounted on a 2D micrometric translation stage to precisely position it, both horizontally and vertically. At the end of the system, we place a grid sheet at $9.5 \mathrm{~cm}$ from lens 1, as main object, while the image is taken using a fixed camera located at $40.8 \mathrm{~cm}$ from lens 5 . In addition to this, the cloaking effect can be also observed with the naked eye and in a wide angular range.

The experimental procedure is as follows: the object is placed at the point where, according to the simulations, there is a maximum concentration of rays. Once the object is in the desired plane, its vertical position is controlled using the translation stage. The distances at which the object becomes visible (height limit) or invisible are checked using a laser telemeter. That height limit $\left(h_{i}\right)$ is then used to measure the spot diameter $\left(d_{i}\right)$ and then the invisible region using the following expression: 


$$
d_{i}=5.48 \mathrm{~cm}-2 \cdot\left(18.5 \mathrm{~cm}-h_{i}\right)
$$

where $i=1,3,4$ are the planes under study, $18.5 \mathrm{~cm}$ is the height of the lenses respect the optical table and $5.48 \mathrm{~cm}$ is the diameter of the lenses, including the lens mount. Once this spot diameter is known, we can calculate the area of invisibility using Equation (10) and compare with the numerical results.

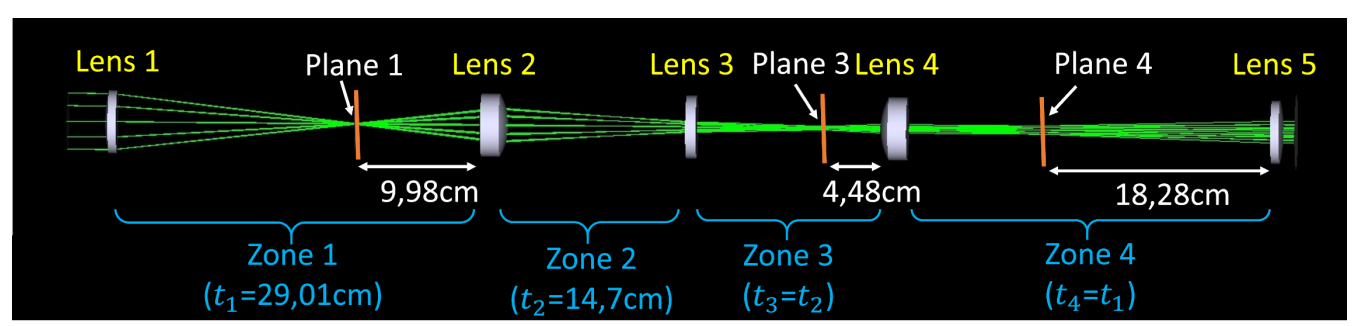

(a)

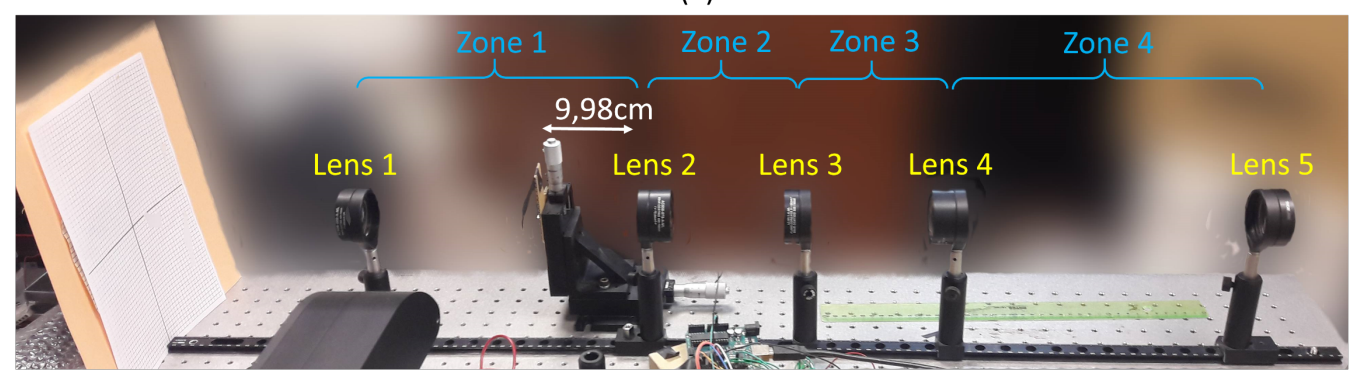

(b)

Figure 3. Case with $f_{3}=-150 \mathrm{~mm}$. (a) Scheme of the ray tracing of the considered system, including the distances between the lenses and the relative position of the invisible planes. (b) Picture of the experimental system for the case $f_{3}=-150 \mathrm{~mm}$.

Although the numerical results show that the best invisible regions are located in planes 1 and 3, we also analyze experimentally plane 4 because, despite the fact that the cloaked area is smaller, it could be interesting in terms of having a larger invisible region in the horizontal axis (see Figure 3a).

Figure 4 shows an example of cloaking the object in plane 1 while the background is perfectly observed. As has been said before, our lenses are at a height of $18.5 \mathrm{~cm}$, and have a diameter of 2" $(D=5.08 \mathrm{~cm})$, and therefore it can be calculated that the axis of the optical system will be at a height of $15.76 \mathrm{~cm}$. This must be taken into account when making the measurements, in order to avoid non physical situations, as those of the invisible objects crossing the optical axis.

The object is placed at $9.98 \mathrm{~cm}$ from lens 2, as it can be seen in Figure $3 \mathrm{~b}$, because it is the point of maximum concentration of rays according to our simulations. First, the object is invisible for the observer, as it can be seen on Figure 4a, proving the proper operation of our system. Then, we lower the object until it begins to be visible (Figure $4 \mathrm{~b}$ ). This corresponds to an object height of $h_{1}=15.8 \mathrm{~cm}$. If we continue lowering the object, it becomes fully visible, as it can be seen in Figure 4c.

Considering that the object becomes experimentally visible at $h_{1}=15.8 \mathrm{~cm}$, the diameter of the spot at this location, using Equation (11), is $d_{1}=0.8 \mathrm{~mm}$ and the area of invisibility in this case is $20.263 \mathrm{~cm}^{2}$. This perfectly matches the numerical results.

Following the same procedure, the area of invisibility in zones 3 and 4 can be calculated. The final results are shown in Table 1. 


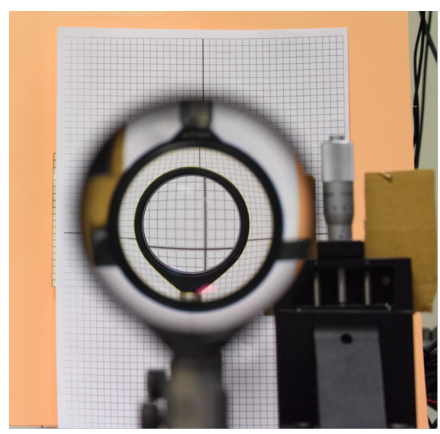

(a)

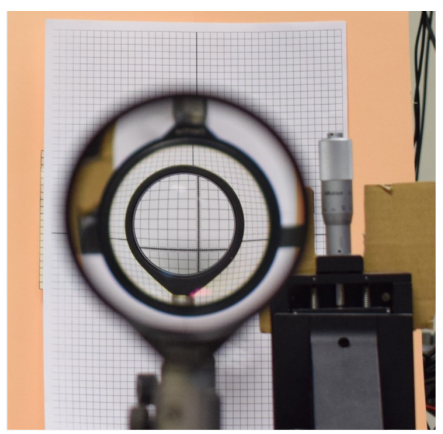

(b)

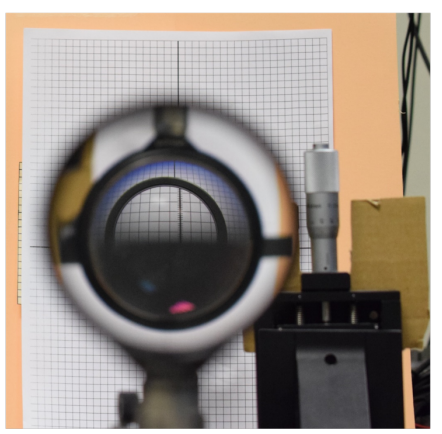

(c)

Figure 4. Photographs taken of the experimental system, in the cases when the object was invisible and when it was not, in the first zone for $f_{3}=-150 \mathrm{~mm}$. (a) Invisible object. (b) Height limit. The object begins to be visible. (c) Visible object.

\subsection{Results for $f_{3}=-25 \mathrm{~mm}$}

As previously mentioned, the proposed theory can be satisfied using different values of the focal length. To show it, here we consider a focal length of the third lens of $-25 \mathrm{~mm}$. Consequently and following the mathematical formalism developed above, the lens positions to achieve invisibility are $t_{1}=t_{4}=332.794 \mathrm{~mm}$ and $t_{2}=t_{3}=122.328 \mathrm{~mm}$. Therefore, the total length of the system is $L=2 t_{1}+2 t_{2}=910.244 \mathrm{~mm}$. The first conclusion about this study is that, although a reduction of the focal length may infer that the total length may be smaller, this is not right. The relationship between the focal lengths and the interlens distances mean that this conclusion cannot be straightforwardly applied. Additionally, from an experimental point of view, a smaller focal length means a smaller lens diameter in several cases. For instance, in this case we should use a lens from Thorlabs with a diameter of $1 / 2^{\prime \prime}(12.7 \mathrm{~mm})$ instead of $2^{\prime \prime}$ as before. However, as it is shown, the system still works.

Following the same procedure as before, the numerical simulation of the ray tracing of this case is shown in Figure 5a. The ray concentration is clearly observed in the spatial regions between lenses 1 and 2 (plane 1) and lenses 4 and 5 (plane 4), providing two invisible regions. There is a third concentration point (plane 3), but it is located just at the position of lens 3 . Thus, it is unpractical experimentally. Nevertheless and following the same procedure as before, Figure $5 \mathrm{~b}$ shows the spot diagram in each plane, including plane 3. The scale is the same to allow a direct comparison.

Analyzing the spot diagram, we can conclude that again plane 1 provides the most ray concentration and then the maximum invisible region. In particular, the results of the spot diameter $\left(d_{i}\right)$ and the invisible regions $\left(A_{i}\right)$ are:

- $\quad$ Plane 1: $d_{1}=0.9864 \mathrm{~mm} \rightarrow A_{1}=20.261 \mathrm{~cm}^{2}$

- $\quad$ Plane 4: $d_{4}=4.598 \mathrm{~mm} \rightarrow A_{4}==20.102 \mathrm{~cm}^{2}$

This means that the invisibility in these planes corresponds to the $99.9 \%$ and $99.2 \%$ of the incident visual field (area of the first and last lenses), respectively. Although any object placed in those regions will be not visible, the effective visual field is practically limited by the area of the smallest lens, this is the third one, as it will be shown in the experiments.

The experimental verification of these results, according to the rays diagram, is shown in Figure 6. As was previously mentioned, lens 3 has a smaller focal length and also a smaller diameter $\left(1 / 2^{\prime \prime}\right)$ because of the commercial limitations. The positions of the lenses are those labeled in Figure 6a, which were calculated using the developed formalism. In this case, the vertical alignment of the optical axis was also carefully adjusted because of the different diameters of the lenses. 


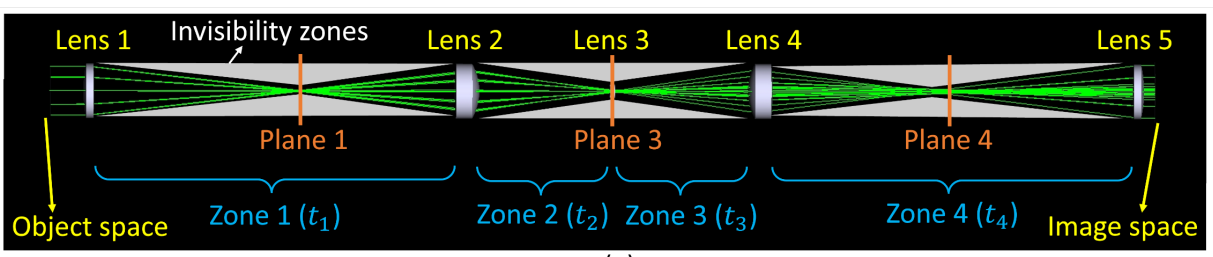

(a)

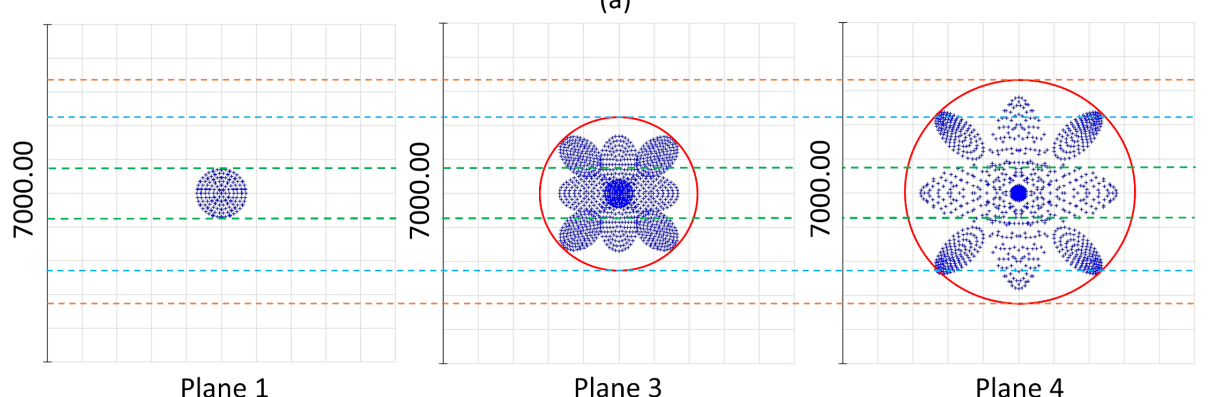

(b)

Figure 5. Case with $f_{3}=-25 \mathrm{~mm}$. (a) Ray tracing for the case $f_{3}=-25 \mathrm{~mm}$. We can see the zones of invisibility and the planes used to calculate the diameter of the beam at the points of maximum invisibility. (b) Spot Diagram for the three planes of interest using the same scale to compare the sizes.

The object was placed in the region of plane 1 because it provides the largest cloaking area in the simulations. In particular, this cloaking region is located at a distance of $14.32 \mathrm{~cm}$ with respect to lens 2 as it can been observed in Figure 6b. As before, the object is a black ruler whose position can be changed using a micrometer. Figure 7 shows two examples where the object is in the invisible region, this is very close to the optical axis but without crossing it (Figure 7a) and in the edge of this region (Figure 7b). Experimentally, we obtained that the area of this invisible region is $20.207 \mathrm{~cm}^{2}$. This result is quite similar to that of the numerical results, validating our methodology.

From a general point of view and following the same procedure, it is possible to calculate the invisible area in all the interesting regions. Table 1 summarizes these results obtained both theoretical and experimentally. Again, the similarity between numerical and experimental results is very high, reinforcing the proposed method. The observed differences are mainly related to the precision of the experimental measurements.

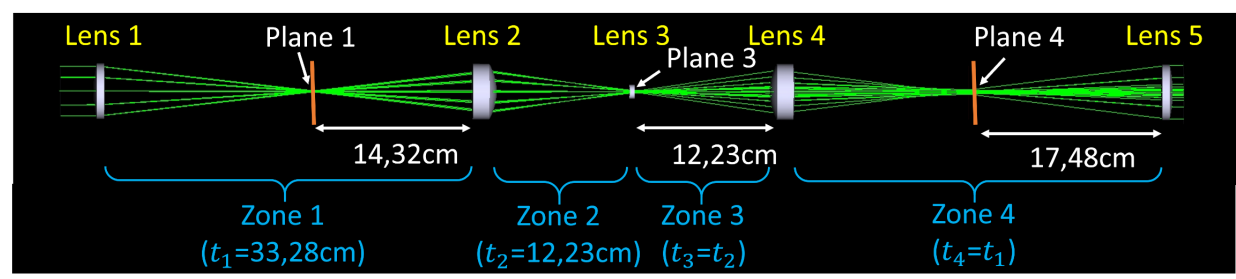

(a)

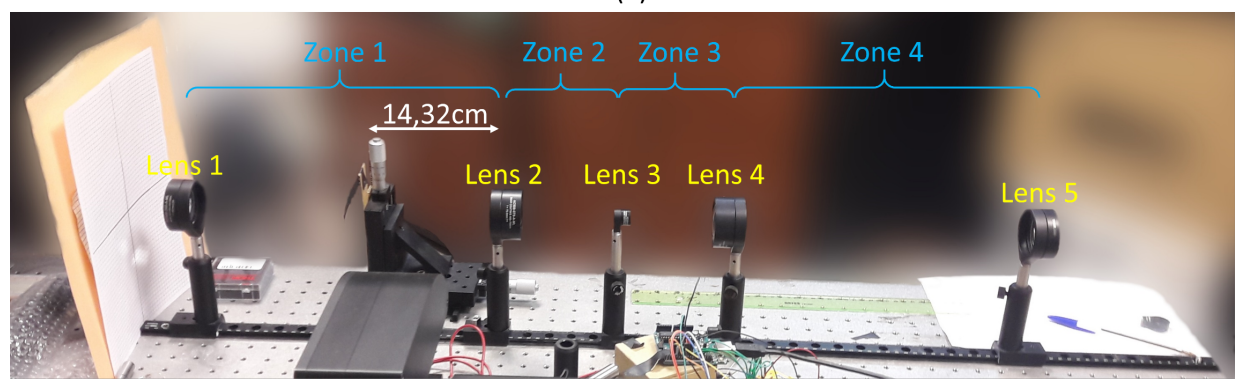

(b)

Figure 6. Case with $f_{3}=-25 \mathrm{~mm}$, simulated and experimental system. (a) Simulation of the ray tracing and zones for the case $f_{3}=-25 \mathrm{~mm}$. (b) Experimental system for the case $f_{3}=-25 \mathrm{~mm}$. 


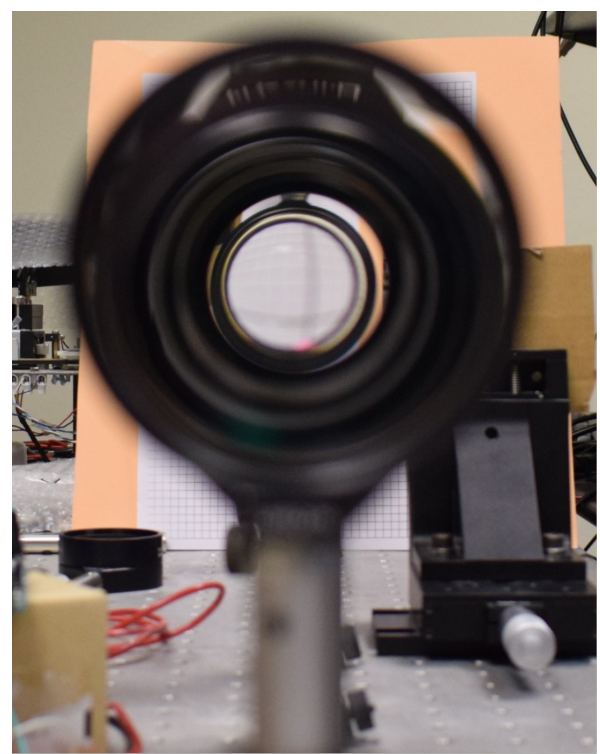

(a)

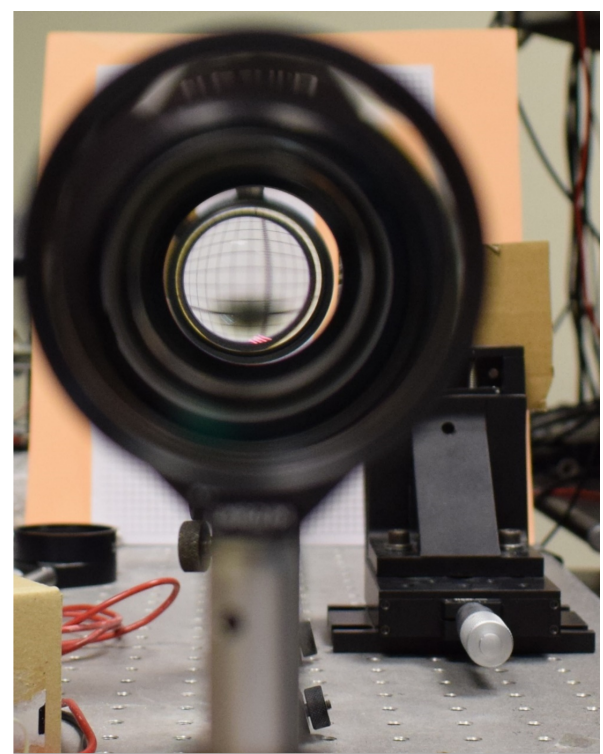

(b)

Figure 7. Photographs taken of the experimental system, in the cases when the object was invisible and when it was not, in the first zone for $f_{3}=-25 \mathrm{~mm}$. (a) Invisible object. (b) Height limit. The object begins to be visible.

Table 1. Comparison between the theoretical and the experimental spot diameters and invisibility areas, for the two cases of interest $\left(f_{3}=-28 \mathrm{~mm}\right.$ and $\left.f_{3}=-150 \mathrm{~mm}\right)$.

\begin{tabular}{|c|c|c|c|c|c|}
\hline & \multirow{2}{*}{$\begin{array}{c}\text { Focal Distance } f_{3} \\
\text { Measurement Form }\end{array}$} & \multicolumn{2}{|c|}{$-150 \mathrm{~mm}$} & \multicolumn{2}{|c|}{$-25 \mathrm{~mm}$} \\
\hline & & Experimental & Theoretical & Experimental & Theoretical \\
\hline \multirow{3}{*}{ Plane 1} & $h_{1}(\mathrm{~mm})$ & 158 & - & 159 & - \\
\hline & Diameter of the spot, $d_{1}(\mathrm{~mm})$ & 0.800 & 0.811 & 2.800 & 0.986 \\
\hline & Invisible Area $\left(\mathrm{mm}^{2}\right)$ & 2026.327 & 2026.314 & 2020.672 & 2026.066 \\
\hline \multirow{3}{*}{ Plane 3} & $h_{3}(\mathrm{~mm})$ & 159 & - & - & - \\
\hline & Diameter of the spot, $d_{3}(\mathrm{~mm})$ & 2.800 & 3.385 & 18.700 & 12.700 \\
\hline & Invisible Area $\left(\mathrm{mm}^{2}\right)$ & 2020.672 & 2017.832 & 1752.184 & 1900.153 \\
\hline \multirow{3}{*}{ Plane 4} & $h_{4}(\mathrm{~mm})$ & 163 & - & 161 & - \\
\hline & Diameter of the spot, $d_{4}(\mathrm{~mm})$ & 10.800 & 6.644 & 6.800 & 4.598 \\
\hline & Invisible Area $\left(\mathrm{mm}^{2}\right)$ & 1935.221 & 1992.160 & 1990.513 & 2010.225 \\
\hline
\end{tabular}

\section{Discussion}

To summarize, this work presents that the cloaking system proposed by Choi et al. [16] can be generalized to a larger number of lenses. This allows the appearance of new invisible planes, increasing the versatility of the optical system and its potential application.

In this sense, we proposed a theoretical formalism considering a system composed of five lenses, that can be extended depending on the requirements. The results of this analytical study determine the required focal lengths and the separation between the lenses. A numerical simulation of the proposed system composed of five lenses revealed that there are three potential invisible planes with different areas of invisibility. While one of the planes present a strong confinement of the light, increasing the invisible area; other plane offers less area but in a larger horizontal range.

We also experimentally verified our results assembling in the lab two different configurations, as representative examples: those considering that $f_{3}=-150 \mathrm{~mm}$ and $f_{3}=-25 \mathrm{~mm}$. The results of the experiments completely agree with the numerical ones showing that we achieved a fully operational invisibility system with five lenses. 
We want to highlight that our main contribution lies in the fact that we demonstrated that this paraxial optical cloaking system is a scalable system, being able to produce more than one invisible region within the optical system by adding new lenses. Consequently, it would be possible to play with the visibility of more than one object using the same system, even making both or only one of them invisible. This could be interesting in imaging manipulation (e.g., holographic technologies) or to hide large objects (e.g., sensors, instrumentation and orbital satellites).

Although the proposed system is able to achieve invisibility using a simple and easy optical system in several regions, it still has relevant limitations for certain applications that would be improved in the future. For instance, as we are considering a ray analysis in the paraxial approximation only the amplitude of the field is completely reproduced while the phase-matching is not warranted. However, the full-field cloaking can be achieved by adding a correction thin field at the beginning or end of the optical system [19].

In a similar way, aberrations (e.g., edge effects) are not taken into account as we are working close to the optical axis (paraxial approximation). A detailed analysis of the image can present these effects related to the edge of the lenses and its correction would be required for certain applications. Finally, the operation region of the system is limited to small angles around the optical axis, excluding the axis. Although the system is properly working in a range of $\pm 30^{\circ}$, it is not able to work in any direction, as an ideal cloaking system. In the literature, we can find several solutions to achieve omnidirectional cloaking [20,21], but with other important limitations, such as the operation bandwidth.

Finally, if the object crosses the optical axis, it will be visible. To avoid this, some mirrors can be added to the proposed system. However, this solution makes the system more complex, and the potential integration problems increase. Consequently, there are still several features that should still be investigated in order to make the system more versatile and closer to an ideal cloaking system.

Author Contributions: Conceptualization, B.G.-C. and J.M.S.-P.; methodology, A.F.-H., B.G.-C. and J.C.T.; software, A.F.-H. and B.G.-C.; validation, A.F.-H. and B.G.-C.; formal analysis, A.F.-H., B.G.-C. and J.C.T.; investigation, A.F.-H., B.G.-C., J.C.T. and J.M.S.-P.; resources, B.G.-C. and J.M.S.-P.; writingoriginal draft preparation, A.F.-H. and B.G.-C.; writing-review and editing, A.F.-H., B.G.-C., J.C.T. and J.M.S.-P.; visualization, A.F.-H., B.G.-C., J.C.T. and J.M.S.-P.; supervision, B.G.-C.; project administration, B.G.-C.; funding acquisition, J.M.S.-P. All authors have read and agreed to the published version of the manuscript.

Funding: This work was supported by the Ministerio de Economía y Competitividad of Spain (Grant number TEC2016-77242-C3-1-R), Spanish Research Agency (Grant number PID2019-109072RB-C31) and Comunidad de Madrid SINFOTON2-CM (Grant number S2018/NMT-4326).These grants are also co-founded by the European Fund for Regional Development.

Institutional Review Board Statement: Not applicable.

Informed Consent Statement: Not applicable.

Data Availability Statement: Not applicable.

Acknowledgments: AF-H wants also to express her grattitude to the Ministerio de Universidades for her predoctoral grant (FPU19/04133).

Conflicts of Interest: The authors declare no conflict of interest.

\section{References}

1. Alù, A. Mantle cloak: Invisibility induced by a surface. Phys. Rev. B 2009, 80, 245115. [CrossRef]

2. Banerjee, D.; Ji, C.; Iizuka, H. Invisibility cloak with image projection capability. Sci. Rep. 2016, 6, 38965. [CrossRef] [PubMed]

3. Chen, H.; Chan, C.T.; Sheng, P. Transformation optics and metamaterials. Nat. Mater. 2010, 9, 387-396. [CrossRef] [PubMed]

4. Shin, D.; Urzhumov, Y.; Jung, Y.; Kang, G.; Baek, S.; Choi, M.; Park, H.; Kim, K.; Smith, D.R. Broadband electromagnetic cloaking with smart metamaterials. Nat. Commun. 2012, 3, 1-8. [CrossRef] [PubMed]

5. Hayran, Z.; Kurt, H.; Herrero, R.; Botey, M.; Staliunas, K.; Staliunas, K. All-dielectric self-cloaked structures. ACS Photonics 2018, 5, 2068-2073. [CrossRef] 
6. Pendry, J.B.; Schurig, D.; Smith, D.R. Controlling electromagnetic fields. Science 2006, 312, 1780-1782. [CrossRef] [PubMed]

7. Leonhardt, U.; Philbin, T.G. General relativity in electrical engineering. New J. Phys. 2006, 8, 247. [CrossRef]

8. Klotz, G.; Mallejac, N.; Guenneau, S.; Enoch, S. Controlling frequency dispersion in electromagnetic invisibility cloaks. Sci. Rep. 2019, 9, 1-7. [CrossRef] [PubMed]

9. Moghbeli, E.; Askari, H.; Forouzeshfard, M. Analyzing the effect of geometric parameters of double split ring resonator on the effective permeability and designing a cloak of invisibility in microwave regime. Appl. Phys. A 2018, 124, 361. [CrossRef]

10. Schurig, D.; Mock, J.J.; Justice, B.; Cummer, S.A.; Pendry, J.B.; Starr, A.F.; Smith, D.R. Metamaterial electromagnetic cloak at microwave frequencies. Science 2006, 314, 977-980. [CrossRef] [PubMed]

11. Chen, X.; Luo, Y.; Zhang, J.; Jiang, K.; Pendry, J.B.; Zhang, S. Macroscopic invisibility cloaking of visible light. Nat. Commun. 2011, 2, 176. [CrossRef] [PubMed]

12. Tsakmakidis, K.; Reshef, O.; Almpanis, E.; Zouros, G.; Mohammadi, E.; Saadat, D.; Sohrabi, F.; Fahimi-Kashani, N.; Etezadi, D.; Boyd, R.; et al. Ultrabroadband 3D invisibility with fast-light cloaks. Nat. Commun. 2019, 10, 1-7. [CrossRef] [PubMed]

13. Zheng, B.; Zhu, R.; Jing, L.; Yang, Y.; Shen, L.; Wang, H.; Wang, Z.; Zhang, X.; Liu, X.; Li, E.; et al. 3D Visible-light invisibility cloak. Adv. Sci. 2018, 5, 1800056. [CrossRef] [PubMed]

14. Islam, S.S.; Faruque, M.R.I.; Hossain, M.J.; Islam, M.T. Limitations of metamaterials for invisibility cloaking. J. Teknol. 2016, 78. [CrossRef]

15. Zhang, B.; Chen, H.; Wu, B.I. Limitations of high-order transformation and incident angle on simplified invisibility cloaks. Opt. Express 2008, 16, 14655-14660. [CrossRef] [PubMed]

16. Choi, J.S.; Howell, J.C. Paraxial ray optics cloaking. Opt. Express 2014, 22, 29465-29478. [CrossRef] [PubMed]

17. Köhler, A. Ein neues Beleuchtungsverfahren für mikrophotographische Zwecke. Zeitschrift für Wissenschaftliche Mikroskopie und für Mikroskopische Technik 1893, 10, 433-440.

18. Hecht, E. Optics, 4th ed.; Addison Wesley: San Francisco, CA, USA, 2002.

19. Choi, J.S.; Howell, J.C. Paraxial full-field cloaking. Opt. Express 2015, 23, 15857-15862. 
[CrossRef] [PubMed]

20. Mitchell-Thomas, R.C.; Sambles, J.; Hibbins, A.P. Omnidirectional surface wave cloak using an isotropic homogeneous dielectric coating. Sci. Rep. 2016, 6, 30984. [CrossRef] [PubMed]

21. Tyc, T.; Oxburg, S.; Cowie, E.N.; Chaplain, G.J.; Macauley, G.; White, C.D.; Courtial, J. Omnidirectional transformation-optics cloak made from lenses and glenses. J. Opt. Soc. Am. A 2016, 33, 1032-1040. [CrossRef] [PubMed] 